\title{
Rolling out HIV antiretroviral therapy in sub-Saharan Africa: 2003-2017
}

\author{
G Taylor ${ }^{1 *}$
}

\section{Abstract}

Multiple issues need to be addressed in order to control the HIV pandemic in sub-Saharan Africa. Combination antiretroviral therapy (cART) is key to reducing morbidity and mortality among people living with HIV and has a role in preventing HIV transmission. However, access to cART is very unevenly distributed globally, especially in sub-Saharan Africa. Although cost of cART is no longer a major barrier as effective treatment can be had for under US\$100 per year, HIV management is compromised by the inadequate number of trained clinicians, the lack of clinical and laboratory infrastructure and the increased prevalence of co-morbidities (e.g., tuberculosis).

To address this disparity, a number of initiatives have been undertaken. One of these was the development of the Infectious Diseases Institute (IDI) at Makerere University, Kampala, Uganda. The goals of the IDI are the clinical care of people living with HIV, clinical research relevant to Uganda (in particular) and sub-Saharan Africa, and clinical training. My initial participation was as a trainer in a program to educate large numbers of clinicians in antiretroviral therapy and other aspects of HIV/AIDS management, with the intention that they become leaders of large clinical programs in their home communities.

Major progress has been made in providing access to CART, and HIV/AIDS mortality and incidence of new cases is decreasing. Nertheless, to reach the World Health Organization 90-90-90 targets by 2020, there remains a need to expand services and develop novel approaches to HIV management. In addition to providing hands-on clinical care, Canadian health care providers can help by transferring clinical skills to local clinicians or by developing streamlined clinical paradigms or new technologies for long-term HIV management in resource-limited settings.

\author{
Affiliation \\ ${ }^{1}$ Department of Medicine, \\ University of Alberta, Edmonton, \\ $A B$
} *Correspondence: geoff.taylor@
ualberta.ca

Suggested citation: Taylor G. Rolling out HIV antiretroviral therapy in sub-Saharan Africa: 2003-2017. Can

Commun Dis Rep. 2018;44(2):68-70. https://doi.org/10.14745/ccdr.v44i02a06

\section{Introduction}

According to the Joint United Nations Programme on HIV/AIDS (UNAIDS), there have been more than 35 million HIV-related deaths since the onset of the HIV/AIDS pandemic in the early 1980s. As of 2016, an estimated 36.7 million people globally were living with HIV, with 25 million living in sub-Saharan Africa. In that year, there were 1.8 million new cases and 1.0 million deaths worldwide (1).

Antiretroviral therapy (ART) has been used for the treatment and prevention of HIV infection since its introduction in the late 1980s. Since the introduction of combination ART (cART) in high-income countries, morbidity and mortality among people living with HIV has been markedly reduced, and CART is currently indicated for all HIV-positive people (2). By suppressing viral replication, cART renders HIV-positive people effectively noninfectious for their intimate contacts. Consequently, pending the development of other biomedical interventions such as an effective vaccine, cART has become an important part of the approach to HIV prevention (2).
In the early 2000s, global access to cART was very unevenly distributed. In his keynote address to the International AIDS Society (IAS) in Durban, South Africa in 2000, then IAS President Mark Wainberg stated that as few as 7,000 individuals in all of sub-Saharan Africa were receiving cART. To address this disparity, in 2001 a group of physicians from the Infectious Diseases Society of America (IDSA) partnered with colleagues at Makerere University, Kampala, Uganda to develop the Infectious Diseases Institute (IDI) at Makerere University (3). The goals of the IDI are the clinical care of people living with HIV, clinical research relevant to sub-Saharan Africa and clinical training.

My initial participation was as a trainer in a program educating large numbers of clinicians in ART and other aspects of HIV/ AIDS management, with the intention that they become leaders of large clinical programs in their home communities. With the development of local expertise, responsibility for training later transferred to local faculty. Since then I became an external examiner for Makerere University, to ensure maintenance of standards. 
Clinical training at IDI involves a combination of didactic learning and clinical experiences with patients in inpatient and outpatient clinics in Kampala and the surrounding rural communities. The principal students, graduate physicians from multiple sub-Saharan countries, attend the training program for a two- to four-week period.

As of 2016, IDI has trained 19,691 health care providers, including physicians, pharmacists, lab technicians and research associates, from 28 African countries (4).

\section{The current Situation}

The following are a number of entirely personal observations on the evolution of an approach to addressing HIV/AIDS management in sub-Saharan Africa.

\section{Access to antiretroviral drugs is no longer a major issue}

Initially, brand-name antiretroviral agents (ARVs) were prohibitively expensive for both patients and public programs in sub-Saharan Africa, costing upwards of US $\$ 10,000$ per year per person (3). A number of workarounds were developed: generic ARVs, produced off patent licence, partially filled the gap; later, brand-name manufacturers licensed generic manufacturers to produce a larger range of ARVs. Currently, cART can be had for as little as US\$100 per year (5). Donors have been crucially important in supporting public programs; primary donors are the Global Fund, currently spending US\$4 billion per year supporting locally run programs that combat HIV/AIDS, tuberculosis and malaria, and the United States President's Emergency Plan for AIDS Relief (PEPFAR; a US government program), which has provided US\$72 billion in HIV/AIDS support since 2003. Together, these and other initiatives have allowed African governments to progressively increase ART availability (1).

\section{Shortage of clinicians trained in HIV management is a huge barrier to treatment}

Uganda, a country of 41 million (versus 36 million in Canada), has four medical schools; there are 17 in Canada. The physician to population ratio is $10: 100,000$ in Uganda, compared with 228:100,000 in Canada. Similarly, the nurse to patient ratios are very low in inpatient units, and patients are expected to have an "attendant," a family member who provides much of the hands-on care.

Most clinicians providing HIV care are generalists and need a straightforward approach to ART. Typically, clinicians use the World Health Organization guidelines (2), which follow a public health approach to therapy rather than more complex specialist guidelines.

Physician salaries are typically very low; even in academia specialists supplement their meagre salaries with after-hours private practice. Salaries offered by non-governmental organizations (NGOs) may be higher than those in the public sector-an important issue for NGOs if they are to avoid distorting the medical labour market by drawing clinicians away from understaffed public programs. Some students in the IDI training programs are seeking certification specifically to make themselves more attractive to recruitment by higher paying NGOs.

\section{Clinical challenges}

Health care providers in sub-Saharan Africa are functioning under major disadvantages compared with those in high-income settings. They have to address challenges associated with very high numbers of HIV-positive patients and growing numbers requiring treatment needing close follow-up. In addition, HIV is only one of the conditions requiring active attention. Tuberculosis infection, together with or separate from HIV, is very common in inpatient units in Uganda, yet sensitive tuberculosis diagnostics are lacking. Negative air pressure hospital rooms are non-existent. Disposable N95 respirators are rare and, when available, are usually reused. As a result, tuberculosis transmission on inpatient units (to patients and staff) has not been uncommon.

In ambulatory settings, lab infrastructure for HIV monitoring, particularly quantitative plasma HIV RNA (i.e. viral load) to assess ART effectiveness, is rarely available (6). Instead, clinicians see patients much more frequently than is the case in Canada, examining them for signs of clinical failure. In so doing, they impose an even greater burden on both patients and clinic infrastructure. When treatment failure occurs, antiretroviral resistance testing is non-existent, so the clinicians rely only on educated guesses about salvage therapy (2). A number of newer ARVs have a much higher barrier to development of resistance (7). These agents are not currently first line therapy in sub-Saharan Africa because of cost; if they could be made available they would provide a potential solution to the problem of antiretroviral drug resistance.

\section{Discussion}

Across sub-Saharan Africa, major strides have been made in expanding ART. In 2015, approximately 17 million of 37 million HIV-positive people worldwide and 12 million in sub-Saharan Africa were receiving treatment (1). HIV/AIDS mortality is falling across the continent. Nevertheless, much is still to be done. In 2016, the total population of Ugandans living with HIV was estimated to be 1.4 million (compared with 75,500 Canadians in 2014), and there were 52,300 new cases (compared with 2,570 in Canada in 2014) $(1,8)$. Each case is a candidate for identification, evaluation, counselling, ART initiation, long-term monitoring and retention in care.

\section{What does the future hold?}

To reach the UNAIDS 90-90-90 target for 2020 (of having $90 \%$ of all people living with HIV knowing their status; $90 \%$ of those diagnosed on antiretroviral treatment; and $90 \%$ of those on antiretroviral treatment virologically suppressed) (2), ART programs across sub-Saharan Africa will need to be greatly expanded. This will need to be accomplished without losing contact with and virologic control in currently treated patients; nor should this be done at the expense of other health care priorities. This is a daunting prospect; it remains to be seen if HIV can become the first viral condition ever controlled without the 
use of an effective vaccine. Novel approaches to management need to be devised, such as initiating ART at first contact. Lab infrastructure needs to be addressed. The CD4 counts no longer have a central role in determining eligibility for treatment. Viral load determination in dried blood spots rather than plasma can greatly increase access to information on viral load, especially in settings far from processing laboratories. Although there is some loss in sensitivity when using dried blood spots, it may be the preferable option given that viral load testing capacity is currently weak across sub-Saharan Africa $(6,9)$.

\section{What can Canadian health care providers do?}

Participating in controlling the pandemic in sub-Saharan Africa and other low resource settings can take different forms. Hands-on direct patient care can be highly satisfying, and extended direct clinical care by Canadian clinicians can make a difference.

For the uninitiated, clinical practice in sub-Saharan Africa is very different from that in Canada: clinics are high volume; investigative resources, even in tertiary care public hospitals, are often lacking. Clinicians often need to adopt a syndromic rather than a diagnostic approach to patient management. Familiarity with a basic but not necessarily subspecialist level of HIV/AIDS management, including CART, is needed, as are skills in management of related complications, particularly tuberculosis. I strongly recommend linking to an existing NGO with a record of accomplishment rather than attempting to develop a new program. Sustainability of any initiative is key to success.

Canadian health care providers can also assist in other ways. Clinicians, especially clinician-educators familiar with HIV/AIDS or related conditions, can transfer clinical skills to local providers. Laboratorians, health system analysts and health policy consultants can advise on developing streamlined clinical paradigms or new technologies for long-term HIV management in resource-limited settings.

Great strides have been made in managing the HIV/AIDS pandemic in sub-Saharan Africa. However, many challenges remain and opportunities still abound for Canadian health care providers to help.

\section{Conflict of interest}

None.

\section{Acknowledgements}

My participation in the Infectious Diseases Institute (IDI) and Makerere University clinical training program has been supported by multiple sources, including the Infectious Diseases Society of America (IDSA), Makerere University, Rotary Club of Edmonton, the University of Alberta and the Accordia Global Health Foundation.

\section{References}

1. UNAIDS data. 2017. Geneva: Joint United Nations Programme on HIV/AIDS (UNAIDS); 2017. http://www. unaids.org/en/resources/documents/2017/2017_data_book

2. World Health Organization. Consolidated guidelines on the use of antiretroviral drugs for treating and preventing HIV infection. Recommendations for a public health approach. 2nd ed. Geneva: World Health Organization; 2016. http:// www.who.int/hiv/pub/arv/arv-2016/en/

3. Ronald A, Kamya M, Katabira E, Scheld WM, Sewankambo $\mathrm{N}$. The Infectious Diseases Institute at Makerere University, Kampala, Uganda. Infect Dis Clin North Am 2011 Jun;25(2):369-83. DOl (http://dx.doi.org/10.1016/j. idc.2011.02.007). PubMed (https://www.ncbi.nlm.nih. gov/entrez/query.fcgi?cmd=Retrieve $\& \mathrm{db}=$ PubMed\&lis t_uids=21628052\&dopt=Abstract).

4. Infectious Diseases Institute. College of Health Sciences, Makerere University: annual report July 2015-June 2016. Kampala (UG): Makerere University; 2016. http://www. idi-makerere.com/docs/IDI\%20Annual\%20Report\%202015. pdf

5. UNAIDS. Press release: HIV treatment now reaching more than 6 million people in sub-Saharan Africa. Geneva: Joint United Nations Programme on HIV/AIDS; 6 July 2012. http:// www.unaids.org/en/resources/presscentre/pressreleaseandst atementarchive/2012/july/20120706prafricatreatment

6. Lecher S, Williams J, Fonjungo PN, Kim AA, Ellenberger $D$, Zhang $G$ et al. Progress with Scale-Up of HIV Viral Load Monitoring - Seven Sub-Saharan African Countries, January 2015-June 2016. MMWR Morb Mortal Wkly Rep 2016 Dec;65(47):1332-5. DOI (http://dx.doi.org/10.15585/ mmwr.mm6547a2). PubMed (https://www.ncbi.nlm.nih. gov/entrez/query.fcgi?cmd=Retrieve\&db=PubMed\&lis t_uids=27906910\&dopt=Abstract).

7. Panel on Antiretroviral Guidelines for Adults and Adolescents. Guidelines for the use of antiretroviral agents in adults and adolescents living with HIV. Washington (DC): US Department of Health and Human Services; 2017. http:// www.aidsinfo.nih.gov/ContentFiles/AdultandAdolescentGL. pdf

8. Public Health Agency of Canada. HIV and AIDS in Canada, Surveillance Report to December 31, 2014. Ottawa (ON): Public Health Agency of Canada; 2015. https:// www.canada.ca/en/public-health/services/publications/ diseases-conditions/hiv-aids-canada-surveillanc e-report-december-31-2014.html

9. Johannessen A, Garrido C, Zahonero N, Sandvik L, Naman E, Kivuyo SL et al. Dried blood spots perform well in viral load monitoring of patients who receive antiretroviral treatment in rural Tanzania. Clin Infect Dis 2009 Sep;49(6):976-81. DOI (http://dx.doi.org/10.1086/605502). PubMed (https://www. ncbi.nlm.nih.gov/entrez/query.fcgi?cmd=Retrieve\&db=PubM ed\&list_uids=19663598\&dopt=Abstract). 